\title{
CONCERNING THE COMMUTATOR SUBGROUPS OF GROUPS WHOSE ORDERS ARE POWERS OF PRIMES.
}

\author{
BY DR. W. B. FITE.
}

( Read before the American Mathematical Society, September 2, 1902.)

In the Transactions of the American Mathematical SoCIETY, volume 3 (1902), pages 331, 351, the writer has shown that the commutators of a metabelian group are invariant in the group, and that those operators of a group which correspond to the invariant operators of the group of cogredient isomorphisms are commutative with all the commutators of the group. It follows as a direct consequence of these two facts that the commutator subgroup of a group of the second or third class is abelian, that is, is of the first class. It was shown in the same article, page 349 , that a metabelian group of odd order cannot be a group of cogredient isomorphisms if it has a set of generators such that the order of any one of them is not a divisor of the least common multiple of all the others. It is the main object of this paper to show that these facts are special cases of more general ones.

Let $G$ be a group of order $p^{m}$ ( $p$ being a prime) and let $G^{\prime}$ be the group of cogredient isomorphisms of $G$, and $G^{\prime \prime}$ that of $G^{\prime}$; also let $l$ and $l^{\prime \prime}$ denote the classes of the commutator subgroups of $G$ and $G^{\prime \prime}$ respectively. If $B_{1}$ is any commutator of $G$, and $A_{i}\left(i=1,2, \cdots, l^{\prime \prime}\right)$ a set of any $l^{\prime \prime}$ commutators (not necessarily distinct) of $G$, we have $A_{i}^{-1} B_{i} A_{i}=B_{i} B_{i+1}$. Now since the commutator subgroup of $G^{\prime \prime}$ is of class $l^{\prime \prime}$ it is evident that $B_{l^{\prime \prime}}^{\prime \prime}$ is invariant in this subgroup $\left(B_{l^{\prime \prime}}^{\prime \prime}\right.$ being that operator of $G^{\prime \prime}$ that corresponds to $\left.B_{l^{\prime \prime}}\right)$. Therefore $B_{l^{\prime \prime}+1}$ is invariant in the commutator subgroup of $G$ and $l \leqq l$ " +1 . We have seen that for groups of classes two or three the commutator subgroups are of class one. We have therefore proved the

THEOREM : If a group $G$ is of order $p^{m}$ ( $p$ being a prime) and class $2 k$ or $2 k+1$, its commutator subgroup is of class $l$, where $l \leqq k$.

Suppose now that $G$ is of class $k$, where $k \leqq p$, and has an abelian commutator subgroup. Let $A_{i}^{\prime}(i=1,2, \ldots, n)$, be a set of generators of $G^{\prime}$ of orders $p^{\alpha_{i}}$ respectively, and let $A_{i}$ be 
an operator of $G$ that corresponds to $A_{i}^{\prime}$. If $B$ is any operator of $G$, we have $A_{i}^{-1} B A_{i}=B t_{1}$, and

$$
A_{i}^{-1} t_{j} A_{i}=t_{j} t_{j+1} \quad(j=1,2,3, \cdots, k-1) .
$$

It follows from this that

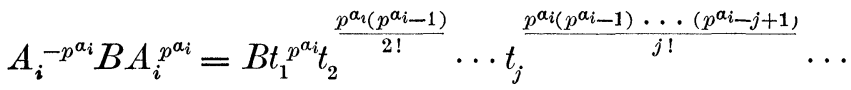

$$
\begin{aligned}
& t_{k-1} \frac{p^{a_{i}\left(p^{a_{i}}-1\right) \cdots\left(p^{a_{i}}-k+2\right)}}{(k-1) !}=B,
\end{aligned}
$$

since $A_{i}^{\prime}$ is of order $p^{a_{i}}$. Also

$$
\begin{aligned}
& A_{i}^{-p^{a_{i}}} t_{j} A_{i}^{p^{a_{i}}}=t_{j} p_{j+1}^{p_{i}} t_{j+2}^{p^{a_{i}\left(p^{a_{i-1}}\right)}} \cdots t_{k-1} \frac{p^{a_{i}\left(p ^ { a _ { i } - 1 ) } \ldots \left(p^{\left.a_{i}-k+j+2\right)}\right.\right.}}{(k-j-1) !}=t_{j}, \\
& (j=1,2, \cdots, k-2)
\end{aligned}
$$

and $t_{j+1}^{p_{i}}=1$, provided $t_{l}^{a_{i}}=1(l>j+1)$. Now $t_{k-1}^{a_{i}}=1$, and therefore $t_{1}^{p^{\alpha_{1}}}=1$.

In a similar way we can show that the order of $t_{1}$ is a divisor of the order of $B^{\prime}$. Also the order of $t_{j+1}(j=1,2, \ldots, k-2)$ is a divisor of the order of $t_{j}^{\prime}$ and therefore a divisor of the order of $B^{\prime}$. It follows from this that if an operator $B$ corresponds to an operator of $G^{\prime}$ of order $p^{\beta}$, then it is commutative with the $p^{\beta}$ th power of every operator of $G$.

Suppose now that $\alpha_{1}>\alpha_{i}(i \geqq 2)$. Then a power of $A_{1}$, less than $p^{a_{1}}$, would be commutative with every operator of $G$. But this is impossible.

THEOREM : If a group $G$, of order $p^{m}$ ( $p$ being a prime) and class $k(k \leqq p)$, has an abelian commutator subgroup, $G^{\prime}$ cannot have a set of generators such that the order of one of them is greater than the order of every one of the others.

We now consider under what conditions a group $G$ of order $p_{1}^{a_{1}} p_{2}^{\alpha_{2}} \cdots p_{n}^{a_{n}}$, where $p_{1}, p_{2}, \cdots, p_{n}$ are distinct primes, can have a commutator subgroup of order $p_{1}^{\beta}$.* Let $G_{1}, G_{2}, \cdots, G_{n}$ be subgroups of $G$ of orders $p_{1}^{\alpha_{1}}, p_{2}^{a_{2}}, \ldots, p_{n}^{a_{n}}$ respectively. Then $G_{i}(i>1)$ is abelian, since identity is the only commutator it contains. Moreover, any operator of $G$ that transforms $G_{i}$ into

* The symmetric group of degree three is such a group. It is of order 3.2 and its commutator subgroup is of order 3 . 
itself is commutative with every operator of $G_{i}$. Let $H_{1}$ be the commutator subgroup. The group $\left\{H_{1}, G_{i}\right\}$ is of order $p_{1}^{\beta} p_{i}^{\alpha_{i}}$. This contains $p_{1}^{\gamma}(\gamma \leqq \beta)$ subgroups of order $p_{i}^{\alpha_{i}}$, and therefore $p_{1}^{\gamma} \equiv 1\left(\bmod p_{i}\right)$. Hence if

$$
p_{1}^{\gamma} \neq 1\left(\bmod p_{i}\right) \quad(0<\gamma \leqq \beta),
$$

every commutator is commutative with every operator of $G_{i}$. Then $A_{j}^{-1} A_{i} A_{j}=A_{i} t_{i}$, where $A_{j}$ is any operator of

$$
G_{j} \quad(j=1,2, \cdots, n)
$$

and $A_{i}$ is any operator of $G_{i}$; and $A_{j}^{-1} A_{i}^{p^{\beta_{i}}} A_{j}=A_{i}^{p^{\beta_{i}}}$, where $p_{1}^{\beta_{i}}$ is the order of $t_{i}$. But $p_{1}^{\beta_{i}}$ is relatively prime to $p_{i}$. Therefore $A_{j}^{-1} A_{i} A_{j}=A_{i}$, and $G$ is the direct product of the groups $G_{j}{ }^{u}$

TheоRem. If a group $G$ of order $p_{1}^{\alpha_{1}} p_{2}^{\alpha_{2}} \cdots p_{n}^{\alpha_{n}}\left(p_{1}, p_{2}, \cdots, p\right.$. being distinct primes) has a commutator subgroup of order $p_{1}^{\beta}$ and if $p_{1}^{\gamma} \neq 1\left(\bmod p_{i}\right)(0<\gamma \leqq \beta),(i=2,3, \cdots n)$, then $G$ is the direct product of groups of orders $p_{1}^{\alpha_{1}}, p_{2}^{\alpha_{2}}, \cdots, p_{n}^{\alpha_{n}}$ respectively.

Cornell University.

August, 1902.

\section{NO'TE ON IRREGULAR DETERMINANTS.}

BY PROFESSOR L. I. HEWES.

Is Gauss's table * of binary quadratic forms the two negative determinants -468 and -931 of the first thousand are classed as regular and their genera and classes given correctly. Perott $\uparrow$ has pointed out that these two determinants are irregular. The details of the classes of the original thirteen irregular determinants of Gauss have been worked out by Cayley : and on the following page are given the details, in his notation, for the properly primitive reduced forms of the two determinants added by Perott's investigation.

* C. F. Gauss, Werke, vol II, p 450.

† "Sur la formation des déterniinants irreguliers," Crelle, vol. 59.

† Cayley's Collected Papers, vol. 5, p. 141. 